\title{
CONTROL IN OBSTACLE-PSEUDOPLATE PROBLEMS WITH FRICTION ON THE BOUNDARY. APPROXIMATE OPTIMAL DESIGN AND WORST SCENARIO PROBLEMS
}

Abstract. In addition to the optimal design and worst scenario problems formulated in a previous paper [3], approximate optimization problems are introduced, making use of the finite element method. The solvability of the approximate problems is proved on the basis of a general theorem of [3]. When the mesh size tends to zero, a subsequence of any sequence of approximate solutions converges uniformly to a solution of the continuous problem.

Introduction. The optimal design problems and reliable solution (worst scenario) problems, which have been introduced and studied in [3], have to be solved approximately. To this end, we employ the simplest kind of finite elements, namely piecewise linear functions over triangulations. In this way the space of state functions and the sets of admissible design variables are discretized in Section 1. To simplify the calculations we also use some quadrature formulae. We define an approximate state problem (variational inequality), optimal design and penalized weight minimization problems. We prove that these problems have at least one solution on the basis of the general Theorem 2.2 of [3]. In Section 2 we study the convergence of approximate solutions when the mesh size tends to zero.

Section 3 is devoted to approximate reliable solutions, i.e., to approximations of the worst scenario method, which has been formulated in [3, Section 4]. We prove the solvability of approximate problems. The convergence of some subsequence of approximate solutions is justified in Section 4 .

2000 Mathematics Subject Classification: 65N30, 74K20, 74P05, 93C30.

Key words and phrases: control of variational inequalities, optimal design, weight minimization, worst scenario, uncertain data. 
1. Approximate optimal design. In the following we use the notation of [3]. Assume that the domain $\Omega$ has a polygonal boundary $\partial \Omega$. Consider a regular family of triangulations $\left\{\mathcal{T}_{h}\right\}, h \rightarrow 0+$, of $\Omega$ which are consistent with all subdomains $\Omega_{i}^{*}$ and $G_{j}$ and with $\partial \Omega_{C}$. We introduce the finite element space of piecewise linear functions

$$
X_{h}=\left\{v_{h} \in C(\bar{\Omega}): v_{h} \mid T \in P_{1}(T) \text { for all triangles } T \in \mathcal{T}\right\}
$$

and the following sets:

$$
\begin{aligned}
V_{h} & =X_{h} \cap V, \\
U_{\mathrm{ad}}^{H h} & =U_{\mathrm{ad}}^{H} \cap X_{h}, \quad U_{\mathrm{ad}}^{\mathcal{Z} h}=U_{\mathrm{ad}}^{\mathcal{Z}} \cap X_{h}^{0}, \quad U_{\mathrm{ad}}^{\mathcal{F} h}=U_{\mathrm{ad}}^{\mathcal{F}} \cap X_{h}^{c},
\end{aligned}
$$

where

$$
\begin{gathered}
X_{h}^{0}=\left.X_{h}\right|_{\bar{\Omega} \backslash \bar{\Omega}^{*}}, \quad X_{h}^{c}=\left.X_{h}\right|_{\partial \Omega_{C}}, \\
U_{\mathrm{ad}}^{h}=U_{\mathrm{ad}}^{H h} \times U_{\mathrm{ad}}^{\mathcal{Z} h} \times U_{\mathrm{ad}}^{\mathcal{F} h}, \\
\mathcal{K}_{h}\left(H_{h}\right)=\left\{v_{h} \in V_{h}: v_{h}(P) \geq H_{h}(P)-O_{i}, i=1, \ldots, N,\right. \\
\left.\quad \text { for all nodes } P \in \Sigma_{h}\right\},
\end{gathered}
$$

where $\Sigma_{h}$ denotes the set of all vertices of triangles $T \in \mathcal{T}_{h}, T \subset \bar{\Omega}^{*}$ and $H_{h} \in U_{\text {ad }}^{H h}$. Note that $\mathcal{K}_{h}\left(H_{h}\right) \subset \mathcal{K}\left(H_{h}\right)$ (cf. [3, (1.3)]).

Let $[p, \xi]_{h}$ be a suitable quadrature formula such that

$$
[p, \cdot]_{h} \in\left(V_{h}\right)^{*}, \quad\left|\langle p, \xi\rangle_{0}-[p, \xi]_{h}\right| \leq C h\|\xi\|_{1}
$$

for all $\xi \in V_{h}$. We define

$$
b_{h}\left(\mathcal{Z}_{h} ; u_{h}, w_{h}\right)=\sum_{T \subset \bar{\Omega} \backslash \Omega^{*} T} \int_{\mathcal{Z}_{h}}\left[u_{h}\right]^{-}(\gamma) w_{h} d x,
$$

where $\gamma$ is the centroid of the triangle $T$ and

$$
\Phi_{h}\left(e_{h}\right)\left(v_{h}\right)=\sum_{E \subset \partial \Omega_{C}} \int_{E} \mathcal{F}_{h}\left|v_{h}(\gamma)\right| d s+I_{\mathcal{K}_{h}\left(H_{h}\right)}\left(v_{h}\right),
$$

where $E$ denotes the edge of a triangle $T \in \mathcal{T}_{h}$ adjacent to $\partial \Omega_{C}$ and $\gamma$ is the midpoint of $E$.

Now we may define the following

Approximate State Problem. Given any $e_{h} \equiv\left\{H_{h}, \mathcal{Z}_{h}, \mathcal{F}_{h}\right\} \in U_{\text {ad }}^{h}$, find $u_{h}\left(e_{h}\right) \in \mathcal{K}_{h}\left(H_{h}\right)$ such that

$$
\begin{aligned}
a\left(H_{h} ; u_{h}\left(e_{h}\right), v_{h}-u_{h}\left(e_{h}\right)\right) & +b_{h}\left(\mathcal{Z}_{h} ; u_{h}\left(e_{h}\right), v_{h}-u_{h}\left(e_{h}\right)\right) \\
& +\Phi_{h}\left(e_{h}\right)\left(v_{h}\right)-\Phi_{h}\left(e_{h}\right)\left(u_{h}\left(e_{h}\right)\right) \\
\geq & {\left[p, v_{h}-u_{h}\left(e_{h}\right)\right]_{h}-2 \omega\left\langle H_{h}, v_{h}-u_{h}\left(e_{h}\right)\right\rangle_{0} }
\end{aligned}
$$

for all $v_{h} \in \mathcal{K}_{h}\left(H_{h}\right)$. 
Finally, let us define the functionals

$$
\begin{aligned}
\mathcal{L}_{\mathrm{DD}}^{h}= & \mathcal{L}_{\mathrm{DD}}, \quad \mathcal{L}_{\mathrm{ISS}}^{h}=\mathcal{L}_{\mathrm{ISS}}, \\
\mathcal{L}_{\mathrm{COM}}^{h}\left(e_{h}, v_{h}\right)= & {\left[p, v_{h}\right]_{h}-2 \omega\left\langle H_{h}, v_{h}\right\rangle_{0}, } \\
\mathcal{L}_{\mathrm{TR}}^{h}\left(e_{h}, v_{h}\right)= & \left\langle H_{h} \operatorname{grad} v_{h}, \operatorname{grad} \theta\right\rangle_{0}+2 \omega\left\langle H_{h}, \theta\right\rangle_{0} \\
& +\sum_{T \subset \bar{\Omega} \backslash \Omega^{*}}\left\langle\mathcal{Z}_{h}\left[v_{h}(\gamma)\right]^{-}, \theta\right\rangle_{0, T}-[p, \theta]_{h} .
\end{aligned}
$$

(Note that the auxiliary function $\theta$ can be chosen in $X(\Omega) \cap V_{h}$.)

We introduce

Approximate Optimal Design Problems. Given a fixed triangulation $\mathcal{T}_{h}$, find

$$
e_{J}^{* h}=\arg \min _{e_{h} \in U_{\mathrm{ad}}^{h}} \mathcal{L}_{J}^{h}\left(e_{h}, u_{h}\left(e_{h}\right)\right)
$$

where $J=\mathrm{DD}$, ISS, COM, TR and $u_{h}\left(e_{h}\right)$ is the solution of the Approximate State Problem (1.4).

Theorem 1.1. (i) The Approximate State Problem (1.4) has a unique solution $u_{h}\left(e_{h}\right)$ for any $e_{h} \in U_{\mathrm{ad}}^{h}$ and any $h$ sufficiently small.

(ii) The Approximate Optimal Design Problem (1.6) has at least one solution for any $J=\mathrm{DD}$, ISS, COM, TR and for any $h$ sufficiently small.

Proof. Let us verify the assumptions of [3, Theorem 2.2], where we set $U_{\mathrm{ad}}:=U_{\mathrm{ad}}^{h}, e:=e_{h}, V:=V_{h}, \mathcal{K}(e):=\mathcal{K}_{h}\left(H_{h}\right)$ and define $A^{h}\left(e_{h}\right)$ : $V_{h} \rightarrow\left(V_{h}\right)^{*}$ by the relation

$$
\left\langle A^{h}\left(e_{h}\right) v_{h}, w_{h}\right\rangle:=a\left(H_{h} ; v_{h}, w_{h}\right)+b_{h}\left(\mathcal{Z}_{h} ; v_{h}, w_{h}\right),
$$

and

$$
\Phi(e):=\Phi_{h}\left(e_{h}\right), \quad\langle f, v\rangle:=[p, v]_{h}, \quad\langle B(e), v\rangle:=-2 \omega\left\langle H_{h}, v\right\rangle_{0} .
$$

Lemma 1.2. For any $H_{h} \in U_{\text {ad }}^{H h}$ the set $\mathcal{K}_{h}\left(H_{h}\right)$ is a closed convex subset of $V_{h}$. If $H_{h n} \in U_{\mathrm{ad}}^{H h}$ and $H_{h n} \rightarrow H_{h}$ as $n \rightarrow \infty$, then

$$
\mathcal{K}_{h}\left(H_{h}\right)=\operatorname{Lim}_{n \rightarrow \infty} \mathcal{K}_{h}\left(H_{h n}\right) .
$$

Proof. The argument is nearly the same as that for [3, Lemma 2.2]. Instead of the function $\vartheta$ we may take $\vartheta_{h} \in V_{h}$ such that $0 \leq \vartheta_{h} \leq 1$ in $\Omega$ and $\vartheta_{h}=1$ in $\Omega^{*}$.

Lemma 1.3. For any $\mathcal{Z}_{h} \subset U_{\text {ad }}^{\mathcal{Z} h}, u_{h}, w_{h} \in V_{h}$,

$$
b_{h}\left(\mathcal{Z}_{h} ; u_{h}, u_{h}-w_{h}\right)-b_{h}\left(\mathcal{Z}_{h} ; w_{h}, u_{h}-w_{h}\right) \geq-C_{1} h\left\|u_{h}-w_{h}\right\|_{1}^{2},
$$

where $C_{1}$ does not depend on $h$ and $\mathcal{Z}_{h}$. 
Proof. The left-hand side of (1.8) is equal to

$$
\begin{aligned}
& \sum_{T} \int_{T} \mathcal{Z}_{h}\left(u_{h}-w_{h}\right)\left(\left[u_{h}\right]^{-}(\gamma)-\left[w_{h}\right]^{-}(\gamma)\right) d x \\
& =\sum_{T}\left\{\int_{T} \mathcal{Z}_{h}\left(u_{h}(\gamma)-w_{h}(\gamma)\right)\left(\left[u_{h}\right]^{-}(\gamma)-\left[w_{h}(\gamma)\right]^{-}\right) d x\right. \\
& \left.\quad+\int_{T} \mathcal{Z}_{h}\left(\left(u_{h}-w_{h}\right)-\left(u_{h}-w_{h}\right)(\gamma)\right)\left(\left[u_{h}\right]^{-}(\gamma)-\left[w_{h}\right]^{-}(\gamma)\right) d x\right\} \\
& =\sum_{T} M_{T}+\sum_{T} \mathcal{R}_{T} .
\end{aligned}
$$

Since

$$
\left(a^{-}-b^{-}\right)(a-b) \geq\left(a^{-}-b^{-}\right)^{2},
$$

the terms $M_{T}$ are non-negative. For brevity, set $v_{h}:=u_{h}-w_{h}$. Using the estimate $\left|a^{-}-b^{-}\right| \leq|a-b|$, which follows from (1.10), we may write

$$
\left|\mathcal{R}_{T}\right|=\left|\left[u_{h}\right]^{-}(\gamma)-\left[w_{h}\right]^{-}(\gamma)\right| \cdot\left|F\left(v_{h}\right)\right| \leq\left|v_{h}(\gamma)\right| \cdot\left|F\left(v_{h}\right)\right|,
$$

where

$$
\begin{gathered}
F\left(v_{h}\right)=\int_{T} \mathcal{Z}_{h}\left(v_{h}-v_{h}(\gamma)\right) d x \\
\left|F\left(v_{h}\right)\right| \leq \mathcal{Z}_{\max } \int_{T}\left|v_{h}-v_{h}(\gamma)\right| d x \leq \mathcal{Z}_{\max } \int_{T} h_{T}\left|\operatorname{grad} v_{h}\right| d x \\
\leq \mathcal{Z}_{\max } h_{T}^{2}\left|v_{h}\right|_{1, T} .
\end{gathered}
$$

Moreover, a standard affine transformation to the reference triangle $\widehat{T}$ yields

$$
|v(\gamma)| \leq C\|\widehat{v}\|_{1, p, \widehat{T}} \leq C h_{T}^{-2 / p}\|v\|_{1, p, T}
$$

for any $p>2$ and

$$
\left\|v_{h}\right\|_{1, p, T} \leq C h_{T}^{-1+2 / p}\left\|v_{h}\right\|_{1,2, T} .
$$

Combining the previous estimates, we obtain

$$
\left|\mathcal{R}_{T}\right| \leq C_{1} h_{T}\left\|v_{h}\right\|_{1,2, T}^{2}
$$

so that

$$
\left|\sum_{T} \mathcal{R}_{T}\right| \leq C_{1} h\left\|v_{h}\right\|_{1,2, \Omega}^{2}=C_{1} h\left\|u_{h}-w_{h}\right\|_{1}^{2} .
$$

Substituting this estimate into (1.9), we arrive at (1.8).

Using $[3,(2.16)]$ and Lemma 1.3, we may write

$$
\begin{aligned}
a\left(H_{h} ; u_{h}\right. & \left.-w_{h}, u_{h}-w_{h}\right)+b_{h}\left(\mathcal{Z}_{h} ; u_{h}, u_{h}-w_{h}\right) \\
& -b_{h}\left(\mathcal{Z}_{h} ; w_{h}, u_{h}-w_{h}\right) \geq\left(C_{F} H_{\min }-C_{1} h\right)\left\|u_{h}-w_{h}\right\|_{1}^{2} .
\end{aligned}
$$


As a consequence, the strong monotonicity of $[3,(2.1)(\mathrm{iii})]$ is satisfied for sufficiently small mesh size $h$.

Next, we have

$$
\begin{aligned}
& \left|b_{h}\left(\mathcal{Z}_{h} ; u_{h}, w\right)-b_{h}\left(\mathcal{Z}_{h} ; v_{h}, w\right)\right| \\
& =\left|\sum_{T} \int_{T} \mathcal{Z}_{h}\left(\left[u_{h}\right]^{-}(\gamma)-\left[v_{h}\right]^{-}(\gamma)\right) w d x\right| \\
& \leq \sum_{T} \mathcal{Z}_{\max } \int_{T}\left|u_{h}(\gamma)-v_{h}(\gamma)\right| \cdot|w| d x \leq \sum_{T} \mathcal{Z}_{\max }\left\|u_{h}-v_{h}\right\|_{0, T}\|w\|_{0, T} \\
& \leq \mathcal{Z}_{\max }\left\|u_{h}-v_{h}\right\|_{0, \Omega}\|w\|_{0, \Omega} .
\end{aligned}
$$

Here we employed the estimate

$$
\left\|\varphi_{h}\right\|_{\infty, T} \leq C h_{T}^{-1}\left\|\varphi_{h}\right\|_{0, T}
$$

for all $\varphi_{h} \in V_{h}$ [1, Thm. 3.2.6].

Using [3, (2.18)] and (1.14), we deduce that the mapping $A^{h}\left(e_{h}\right)$ from (1.7) is Lipschitz-continuous in $V_{h}$, uniformly in $U_{\text {ad }}$.

Next, let $e_{h n} \rightarrow e_{h}$ as $n \rightarrow \infty, e_{h n} \in U_{\text {ad }}^{h}$. We may write

$$
\begin{aligned}
& \left|\left\langle A^{h}\left(e_{h n}\right) v_{h}-A^{h}\left(e_{h}\right) v_{h}, w\right\rangle\right| \\
\leq & \left\|H_{h n}-H_{h}\right\|_{\infty}\left\|v_{h}\right\|_{1}\|w\|_{1}+\sum_{T}\left\|\mathcal{Z}_{h n}-\mathcal{Z}_{h}\right\|_{\infty}\left\|v_{h}\right\|_{\infty, T} h_{T}\|w\|_{0,2, T} \\
\leq & C\left\|e_{h n}-e_{h}\right\|_{\infty}\left\|v_{h}\right\|_{1}\|w\|_{1},
\end{aligned}
$$

arguing as in the derivation of (1.14). As a consequence,

$$
A^{h}\left(e_{h n}\right) v_{h} \rightarrow A^{h}\left(e_{h}\right) v_{h} \quad \text { in }\left(V_{h}\right)^{*} \text { for all } v_{h} \in V_{h} .
$$

Lemma 1.4. The system of functionals $\left\{\Phi_{h}\left(e_{h}\right)\right\}, e_{h} \in U_{\mathrm{ad}}^{h}$, defined by (1.3), satisfies the assumptions [3, (2.2), (2.3)].

Proof. We can proceed as in the proof of [3, Lemma 2.4]. Write

$$
\phi_{h}\left(e_{h}\right)=\phi_{h}^{(1)}\left(e_{h}\right)+\phi_{h}^{(2)}\left(e_{h}\right),
$$

where

$$
\phi_{h}^{(1)}\left(e_{h}\right) v=\sum_{E \subset \partial \Omega_{C}} \int_{E} \mathcal{F}_{h}|v(\gamma)| d s, \quad \phi_{h}^{(2)}\left(e_{h}\right) v=I_{\mathcal{K}_{h}\left(H_{h}\right)}(v) .
$$

We shall verify the condition [3, (2.2)] by means of [3, Definition 2.2]. Consider a sequence $\left\{e_{h n}\right\}, e_{h n} \rightarrow e_{h}$ as $n \rightarrow \infty, e_{h n} \in U_{\text {ad }}^{h}$.

(i) Let $v_{h} \in \mathcal{K}_{h}\left(H_{h}\right)$. By Lemma 1.2 there exists a sequence $\left\{v_{h n}\right\}$, $v_{h n} \in \mathcal{K}_{h}\left(H_{h n}\right)$, such that $v_{h n} \rightarrow v_{h}$ as $n \rightarrow \infty$. Then 
where

$$
\left|\phi_{h}\left(e_{h n}\right) v_{h n}-\phi_{h}\left(e_{h}\right) v_{h}\right| \leq\left|\lambda_{1 n}\right|+\left|\lambda_{2 n}\right|,
$$

$$
\begin{aligned}
&\left|\lambda_{1 n}\right|=\left|\phi_{h}^{(1)}\left(e_{h n}\right) v_{h n}-\phi_{h}^{(1)}\left(e_{h}\right) v_{h}\right| \\
& \leq\left|\phi_{h}^{(1)}\left(e_{h n}\right) v_{h n}-\phi_{h}^{(1)}\left(e_{h}\right) v_{h n}\right|+\left|\phi_{h}^{(1)}\left(e_{h}\right) v_{h n}-\phi_{h}^{(1)}\left(e_{h}\right) v_{h}\right| \\
& \leq \sum_{E}\left(\left|v_{h n}(\gamma)\right| \int_{E}\left|\mathcal{F}_{h n}-\mathcal{F}_{h}\right| d s+\left|v_{h n}(\gamma)-v_{h}(\gamma)\right| \int_{E} \mathcal{F}_{h} d s\right) \rightarrow 0 \\
& \text { as } n \rightarrow \infty,
\end{aligned}
$$

$\left|\lambda_{2 n}\right|=\left|I_{\mathcal{K}_{h}\left(H_{h n}\right)}\left(v_{h n}\right)-I_{\mathcal{K}_{h}\left(H_{h}\right)}\left(v_{h}\right)\right|=0 \quad$ for all $n$.

Altogether, we have

$$
\lim _{n \rightarrow \infty} \Phi_{h}\left(e_{h n}\right) v_{h n}=\Phi_{h}\left(e_{h}\right) v_{h} .
$$

Second, let $v_{h} \notin \mathcal{K}_{h}\left(H_{h}\right)$. Setting $v_{h n}=v_{h}$ for all $n=1,2, \ldots$, we have

$$
\begin{aligned}
\limsup \Phi_{h}\left(e_{h n}\right) v_{h n} & \leq \limsup \sum_{E} \int_{E} \mathcal{F}_{h n}\left|v_{h}(\gamma)\right| d s+\infty \\
& =\sum_{E} \int_{E} \mathcal{F}_{h}\left|v_{h}(\gamma)\right| d s+\Phi_{h}^{(2)}\left(e_{h}\right) v_{h}=\Phi_{h}\left(e_{h}\right) v_{h} .
\end{aligned}
$$

Combining (1.17) and (1.18), we obtain

$$
\limsup _{n \rightarrow \infty} \Phi_{h}\left(e_{h n}\right) v_{h n} \leq \Phi_{h}\left(e_{h}\right) v_{h} .
$$

(ii) Let $v_{h n} \rightarrow v_{h}$ as $n \rightarrow \infty$. We have

$$
\liminf \Phi_{h}\left(e_{h n}\right) v_{h n} \geq \liminf \phi_{h}^{(1)}\left(e_{h n}\right) v_{h n}+\liminf \phi_{h}^{(2)}\left(e_{h n}\right) v_{h n} .
$$

Arguing as in the case of $\lambda_{1 n}$, we obtain

$$
\lim \Phi_{h}^{(1)}\left(e_{h n}\right) v_{h n}=\Phi_{h}^{(1)}\left(e_{h}\right) v_{h} .
$$

Next, we may write

$$
\liminf I_{\mathcal{K}_{h}\left(H_{h n}\right)}\left(v_{h n}\right)=a,
$$

where $a$ is either $+\infty$ or zero. If $a=+\infty$, then obviously

$$
a \geq I_{\mathcal{K}_{h}\left(H_{h}\right)}\left(v_{h}\right) \text {. }
$$

If $a=0$, there exists a subsequence $\left\{v_{h k}\right\} \subset\left\{v_{h n}\right\}$ such that $v_{h k} \in \mathcal{K}_{h}\left(H_{h k}\right)$ for all $k \rightarrow \infty$. By Lemma 1.2 the limit $v_{h}$ belongs to $\mathcal{K}_{h}\left(H_{h}\right)$, so that $I_{\mathcal{K}_{h}\left(H_{h}\right)}\left(v_{h}\right)=0$ and (1.19) holds again. As a consequence,

$$
\liminf \Phi_{h}^{(2)}\left(e_{h n}\right) v_{h n} \geq \Phi_{h}^{(2)}\left(e_{h}\right) v_{h}
$$

and the condition $[3,(2.2)$ (ii)] is fulfilled.

To satisfy condition $[3,(2.3)]$, we can choose $a_{n}=0$ for all $n$, since $0 \in \mathcal{K}_{h}\left(H_{h n}\right)$ for all $H_{h n} \in U_{\mathrm{ad}}^{H h}$, due to [3, (1.1)]. Then

$$
\Phi_{h}\left(e_{h n}\right) a_{n}=0 \quad \text { for all } n \text {. }
$$


Lemma 1.5. The functionals $\mathcal{L}_{\mathrm{DD}}^{h}, \mathcal{L}_{\mathrm{ISS}}^{h}, \mathcal{L}_{\mathrm{COM}}^{h}, \mathcal{L}_{\mathrm{TR}}^{h}$ satisfy condition $[3,(2.5)]$.

Proof. The proof of the cases $\mathcal{L}_{\mathrm{DD}}^{h}$ and $\mathcal{L}_{\text {ISS }}^{h}$ is the same as that for [3, Lemma 2.5]. Let $e_{h n} \rightarrow e_{h}$ and $v_{h n} \rightarrow v_{h}$ as $n \rightarrow \infty$. We may write

$$
\begin{gathered}
\mathcal{L}_{\mathrm{COM}}^{h}\left(e_{h n}, v_{h n}\right)=\left[p, v_{h n}\right]_{h}-2 \omega\left\langle H_{h}, v_{h n}\right\rangle_{0}+\psi_{n}, \\
\left|\psi_{n}\right|=2 \omega\left|\left\langle H_{h n}-H_{h}, v_{h n}\right\rangle_{0}\right| \leq C\left\|H_{h n}-H_{h}\right\|_{\infty}\left\|v_{h n}\right\|_{0} \rightarrow 0 .
\end{gathered}
$$

Then

$$
\lim _{n \rightarrow \infty} \mathcal{L}_{\mathrm{COM}}^{h}\left(e_{h n}, v_{h n}\right)=\left[p, v_{h}\right]_{h}-2 \omega\left\langle H_{h}, v_{h}\right\rangle_{0}=\mathcal{L}_{\mathrm{COM}}^{h}\left(e_{h}, v_{h}\right) .
$$

Next, we may write

$$
\begin{gathered}
\mathcal{L}_{\mathrm{TR}}^{h}\left(e_{h n}, v_{h n}\right)=\mathcal{L}_{\mathrm{TR}}^{h}\left(e_{h}, v_{h n}\right)+M_{n}, \\
\left|M_{n}\right|=\mid\left\langle\left(H_{h n}-H_{h}\right) \operatorname{grad} v_{h n}, \operatorname{grad} \theta\right\rangle_{0}+2 \omega\left\langle H_{h n}-H_{h}, \theta\right\rangle_{0} \\
+\sum_{T}\left\langle\left(\mathcal{Z}_{h n}-\mathcal{Z}_{h}\right)\left[v_{h n}(\gamma)\right]^{-}, \theta\right\rangle_{0, T} \mid \\
\leq\left(\left\|H_{h n}-H_{h}\right\|_{\infty}+\left\|\mathcal{Z}_{h n}-\mathcal{Z}_{h}\right\|_{\infty}\right)\left(C_{1}\left\|v_{h n}\right\|_{1}+C_{2}\right) \rightarrow 0,
\end{gathered}
$$

using also estimate (1.15) in the last inequality. Making use of (1.15) again, we obtain

$$
\begin{aligned}
\mid \mathcal{L}_{\mathrm{TR}}^{h}\left(e_{h}, v_{h n}\right) & -\mathcal{L}_{\mathrm{TR}}^{h}\left(e_{h}, v_{h}\right) \mid \leq H_{\max }\left\|v_{h n}-v_{h}\right\|_{1}\|\theta\|_{1} \\
& +\mathcal{Z}_{\max } \sum_{T}\left\|v_{h n}-v_{h}\right\|_{0, T}\|\theta\|_{0, T} \leq C\left\|v_{h n}-v_{h}\right\|_{1} \rightarrow 0 .
\end{aligned}
$$

Combining (1.20)-(1.22), we arrive at

$$
\lim _{n \rightarrow \infty} \mathcal{L}_{\mathrm{TR}}^{h}\left(e_{h n}, v_{h n}\right)=\mathcal{L}_{\mathrm{TR}}^{h}\left(e_{h}, v_{h}\right)
$$

We define the following

$$
\text { Approximate Weight Minimization Problem. Find }
$$

$$
e_{h}^{\varepsilon}=\arg \min _{e_{h} \in U_{\mathrm{ad}}^{h}} \mathcal{L}_{W}\left(\varepsilon ; e_{h}, u_{h}\left(e_{h}\right)\right),
$$

where $\mathcal{L}_{W}$ is the penalized cost functional, defined in [3, Section 3].

Theorem 1.6. The Approximate Weight Minimization Problem (1.23) has at least one solution for any positive $\varepsilon$ and any $h$ sufficiently small.

Proof. In proving Theorem 1.1 we have verified all assumptions of the abstract [3, Theorem 2.1], so that

$$
u_{h}\left(e_{h n}\right) \rightarrow u_{h}\left(e_{h}\right)
$$

provided $h$ is sufficiently small, $e_{h n} \in U_{\mathrm{ad}}^{h}$ and $e_{h n} \rightarrow e_{h}$ as $n \rightarrow \infty$. Then the functions

$$
e_{h} \mapsto\left[F_{j}\left(u_{h}\left(e_{h}\right)\right]^{+}, \quad j=1, \ldots, M,\right.
$$


are continuous in $U_{\text {ad }}^{h}$ (cf. the analogous proof of [3, Lemma 3.1]). Since the weight $\left\langle\omega, H_{h}\right\rangle_{0}$ is continuous in $U_{\text {ad }}^{h}$ as well, we find that

$$
e_{h} \mapsto \mathcal{L}_{W}\left(\varepsilon ; e_{h}, u_{h}\left(e_{h}\right)\right)
$$

is continuous in the compact set $U_{\mathrm{ad}}^{h}$. As a consequence, a minimizer $e_{h}^{\varepsilon}$ exists.

2. Convergence results. In the present section we will study the convergence of finite element approximations when the mesh size tends to zero. To this end we establish the crucial

Proposition 2.1. Let $e_{h} \in U_{\mathrm{ad}}^{h}$ with $e_{h} \rightarrow e$ in $U$ as $h \rightarrow 0+$. Then

$$
u_{h}\left(e_{h}\right) \rightarrow u(e) \quad \text { in } V \text { as } h \rightarrow 0+\text {. }
$$

Proof. For brevity, set $u_{h}:=u_{h}\left(e_{h}\right)$. Substituting $v_{h}=0$ in the inequality (1.4) and using (1.1), (1.7), (1.13), we obtain

$$
\begin{aligned}
\left(C_{F} H_{\min }-C_{1} h\right)\left\|u_{h}\right\|_{1}^{2} & \leq\left\langle A^{h}\left(e_{h}\right) u_{h}, u_{h}\right\rangle \leq-\left[p, u_{h}\right]_{h}+2 \omega\left\langle H_{h}, u_{h}\right\rangle_{0} \\
& \leq C_{2}\left\|u_{h}\right\|_{1},
\end{aligned}
$$

so that $\left\|u_{h}\right\|_{1} \leq C$ for all $h$ sufficiently small. As a consequence, there exist $u \in V$ and a subsequence of $\left\{u_{h}\right\}$ (denoted by the same symbol) such that

$$
u_{h} \rightarrow u \quad \text { (weakly) in } V \text {. }
$$

One can prove that $u \in \mathcal{K}(H)$. Indeed, following [2, pp. 33-34], consider any function $\varphi \in C_{0}^{\infty}\left(\Omega_{i}^{*}\right)$ with $\varphi \geq 0$ and define a piecewise constant function

$$
\varphi_{h}=\sum_{T \subset \bar{\Omega}_{i}^{*}} \varphi(\gamma) \chi_{T}
$$

where $\chi_{T}$ is the characteristic function of the triangle $T$ and $\gamma$ is the centroid of $T$. Define $\psi=H-O_{i}$ on $\Omega_{i}^{*}$ and $\psi_{h}=H_{h}-O_{i}$. Then

$$
\lim _{h \rightarrow 0} \int_{\Omega_{i}^{*}}\left(u_{h}-\psi_{h}\right) \varphi_{h} d x=\int_{\Omega_{i}^{*}}(u-\psi) \varphi d x,
$$

since $u_{h} \rightarrow u$ in $L^{2}\left(\Omega_{i}^{*}\right)$ by Rellich's Theorem and $\varphi_{h} \rightarrow \varphi, \psi_{h} \rightarrow \psi$ in $L^{2}\left(\Omega_{i}^{*}\right)$.

On the other hand, we have

$$
\int_{\Omega_{i}^{*}}\left(u_{h}-\psi_{h}\right) \varphi_{h} d x=\sum_{T \subset \bar{\Omega}_{i}^{*}} \varphi(\gamma) \int_{T}\left(u_{h}-\psi_{h}\right) d x .
$$

By definition of $\mathcal{K}_{h}\left(H_{h}\right)$, we obtain

$$
\int_{T}\left(u_{h}-\psi_{h}\right) d x=\frac{1}{3}(\operatorname{meas} T) \sum_{j=1}^{3}\left(u_{h}-\psi_{h}\right)\left(a_{j}\right) \geq 0,
$$


where $a_{j}$ are the vertices of $T$. Combining (2.3) and (2.4), we arrive at

$$
\int_{\Omega_{i}^{*}}\left(u_{h}-\psi_{h}\right) \varphi_{h} d x \geq 0
$$

Then (2.2) yields

$$
\int_{\Omega_{i}^{*}}(u-\psi) \varphi d x \geq 0
$$

which in turn implies that $u \geq \psi$ a.e. in $\Omega_{i}^{*}$, i.e., $u \in \mathcal{K}(H)$.

Next, let us verify that $u$ coincides with a solution $u(e)$ of the variational inequality $[3,(1.7)]$. Consider an arbitrary $v \in \mathcal{K}(H)$. There exists a function $\psi \in C^{(0), 1}(\bar{\Omega})$ such that $\psi=0$ on $\partial \Omega_{D}, \psi=H-O_{i}$ on $\Omega_{i}^{*}$ for all $i=$ $1, \ldots, N$. Then

$$
\omega:=v-\psi \in \mathcal{K}_{0}=\left\{w \in V: w \geq 0 \text { a.e. in } \Omega^{*}\right\} .
$$

Let us employ a regularization operator $\varrho_{\kappa}$ (see e.g. [4]). Let $\varrho_{\kappa} E \psi$ and $\varrho_{\kappa} E \omega$ denote the regularization applied to a proper extension of the functions $\psi$ and $\omega$ to a larger domain $\widetilde{\Omega} \supset \bar{\Omega}$, so that

$$
\varrho_{\kappa} E \omega \geq 0 \quad \text { and } \quad \varrho_{\kappa} E O_{i}=O_{i} \quad \text { on } \Omega_{i}^{*}, i=1, \ldots, N .
$$

We define

$$
v_{h}=\pi_{h}\left(\varrho_{\kappa} E \psi+\varrho_{\kappa} E \omega+\left(\left\|\varrho_{\kappa} E H-H\right\|_{\infty, \Omega^{*}}+\left\|H-H_{h}\right\|_{\infty, \Omega^{*}}\right) \vartheta\right),
$$

where $\vartheta \in C_{0}^{\infty}(\Omega)$ is such that $0 \leq \vartheta \leq 1$ in $\Omega$ and $\vartheta=1$ for $x \in \Omega^{*}$ and $\pi_{h}$ denotes the Lagrange linear interpolation over $\mathcal{T}_{h}$. Consequently, $v_{h} \in V_{h}$ and for any node $P \in \Sigma_{h}$ we have

$v_{h}(P) \geq \varrho_{\kappa} E \psi(P)+\left|\varrho_{\kappa} E H(P)-H(P)\right|+\left|H(P)-H_{h}(P)\right| \geq H_{h}(P)-O_{i}$, so that $v_{h} \in \mathcal{K}_{h}\left(H_{h}\right)$. Furthermore, we may write

$$
\begin{aligned}
\left\|v_{h}-v\right\|_{1}= & \| \pi_{h}\left(\varrho_{\kappa} E \psi\right)-\psi+\pi_{h}\left(\varrho_{\kappa} E \omega\right)-\omega \\
& +\left(\left\|\varrho_{\kappa} E H-H\right\|_{\infty, \Omega^{*}}+\left\|H-H_{h}\right\|_{\infty, \Omega^{*}}\right) \pi_{h} \vartheta \|_{1} \\
\leq & \left\|\pi_{h}\left(\varrho_{\kappa} E \psi\right)-\varrho_{\kappa} E \psi\right\|_{1}+\left\|\varrho_{\kappa} E \psi-\psi\right\|_{1} \\
& +\left\|\pi_{h}\left(\varrho_{\kappa} E \omega\right)-\varrho_{\kappa} E \omega\right\|_{1}+\left\|\varrho_{\kappa} E \omega-\omega\right\|_{1} \\
& +\left(\left\|\varrho_{\kappa} E H-H\right\|_{\infty, \Omega^{*}}+\left\|H-H_{h}\right\|_{\infty, \Omega^{*}}\right)\left\|\pi_{h} \vartheta\right\|_{1} \rightarrow 0
\end{aligned}
$$

as $\kappa \rightarrow 0+$ and $h \rightarrow 0+$.

Here we have used the fact that $H \in W^{1, p}(\Omega)$ for any $p>2$ and

$$
\left\|\varrho_{\kappa} E H-H\right\|_{\infty, \Omega^{*}} \leq C\left\|\varrho_{\kappa} E H-H\right\|_{1, p, \Omega} \rightarrow 0
$$

as $\kappa \rightarrow 0+$ (see [4, Thms. 2.1 and 3.1]).

For any $e_{h} \in U_{\text {ad }}^{h}, u_{h} \in V_{h}$ and $v \in V$ the following estimate holds (see $[3,(2.15)]$ for the definition of $\left.A\left(e_{h}\right)\right)$ :

$$
\left|\left\langle A^{h}\left(e_{h}\right) u_{h}, v\right\rangle-\left\langle A\left(e_{h}\right) u_{h}, v\right\rangle\right| \leq C h\left\|u_{h}\right\|_{1}\|v\|_{0} .
$$


Indeed, we have

$$
\begin{aligned}
\mid\left\langle A^{h}\left(e_{h}\right) u_{h}, v\right\rangle- & \left\langle A\left(e_{h}\right) u_{h}, v\right\rangle|=| b_{h}\left(\mathcal{Z}_{h} ; u_{h}, v\right)-b\left(\mathcal{Z}_{h} ; u_{h}, v\right) \mid \\
& =\left|\sum_{T \subset \bar{\Omega} \backslash \Omega^{*} T} \int_{T} \mathcal{Z}_{h}\left(\left[u_{h}\right]^{-}-\left[u_{h}\right]^{-}(\gamma)\right) v d x\right| \\
& \leq \sum_{T} \int_{T} \mathcal{Z}_{h}\left|u_{h}-u_{h}(\gamma)\right| \cdot|v| d x \leq C h\left\|u_{h}\right\|_{1}\|v\|_{0},
\end{aligned}
$$

arguing as in the proof of Lemma 1.3.

Let us substitute $v_{h}$ in the inequality (1.4) and pass to $\lim \inf$ as $h \rightarrow 0+$. It is easy to see that

$$
\lim \inf \left\langle A(e) u_{h}, u_{h}\right\rangle \geq\langle A(e) u, u\rangle .
$$

In fact, the functional $u \mapsto a(H ; u, u)$ is weakly lower semicontinuous, being convex and differentiable. Second, we may write

$$
\begin{aligned}
\left|b\left(\mathcal{Z} ; u_{h}, u_{h}\right)-b(\mathcal{Z} ; u, u)\right| & \leq \int_{\Omega \backslash \Omega^{*}} \mathcal{Z}\left|u_{h}\left[u_{h}\right]^{-}-u[u]^{-}\right| d x \\
& \leq C \mathcal{Z}_{\max }\left(\left\|u_{h}\right\|_{0}+\|u\|_{0}\right)\left\|u_{h}-u\right\|_{0} \rightarrow 0
\end{aligned}
$$

due to Rellich's Theorem. Hence,

$$
\begin{aligned}
\liminf \left\{a\left(H ; u_{h}, u_{h}\right)+b\left(\mathcal{Z} ; u_{h}, u_{h}\right)\right\} & \geq a(H ; u, u)+b(\mathcal{Z} ; u, u) \\
& =\langle A(e) u, u\rangle .
\end{aligned}
$$

Making use of [3, (2.1)(iv) and Lemma 2.3], we derive that

$$
\left|\left\langle A\left(e_{h}\right) u_{h}, u_{h}\right\rangle-\left\langle A(e) u_{h}, u_{h}\right\rangle\right| \leq\left\|A\left(e_{h}\right) u_{h}-A(e) u_{h}\right\|_{*}\left\|u_{h}\right\|_{1} \rightarrow 0 .
$$

Therefore,

$$
\lim \inf \left\langle A\left(e_{h}\right) u_{h}, u_{h}\right\rangle \geq \lim \inf \left\langle A(e) u_{h}, u_{h}\right\rangle \geq\langle A(e) u, u\rangle
$$

by $(2.10)$ and $(2.11)$.

Making also use of (2.7), we obtain

$$
\begin{aligned}
\lim \inf & \left\langle A^{h}\left(e_{h}\right), u_{h}, u_{h}\right\rangle \geq \liminf \left\langle A\left(e_{h}\right), u_{h}, u_{h}\right\rangle \\
& +\liminf \left(\left\langle A^{h}\left(e_{h}\right) u_{h}, u_{h}\right\rangle-\left\langle A\left(e_{h}\right) u_{h}, u_{h}\right\rangle\right) \geq\langle A(e) u, u\rangle .
\end{aligned}
$$

Next, we prove that

$$
\lim \left\langle A^{h}\left(e_{h}\right) u_{h}, v\right\rangle=\langle A(e) u, v\rangle
$$

for all $v \in V$. Indeed, if we employ (2.7), it suffices to show that

$$
\lim \left\langle A\left(e_{h}\right) u_{h}, v\right\rangle=\langle A(e) u, v\rangle .
$$

First, we may write

$$
\left|\left\langle A\left(e_{h}\right) u_{h}, v\right\rangle-\left\langle A(e) u_{h}, v\right\rangle\right| \leq\left\|A\left(e_{h}\right) u_{h}-A(e) u_{h}\right\|_{*}\|v\|_{1} \rightarrow 0
$$

by $[3,(2.1)($ iv) and Lemma 2.3]. Second, 


$$
a\left(H ; u_{h}, v\right) \rightarrow a(H ; u, v)
$$

by the weak convergence (2.1). Third, we have

$$
\begin{aligned}
\left|b\left(\mathcal{Z} ; u_{h}, v\right)-b(\mathcal{Z} ; u, v)\right| & =\left|\int_{\Omega \backslash \Omega^{*}} \mathcal{Z}\left(\left[u_{h}\right]^{-}-[u]^{-}\right) v d x\right| \\
& \leq \mathcal{Z}_{\max }\left\|u_{h}-u\right\|_{0}\|v\|_{0} \rightarrow 0 .
\end{aligned}
$$

Then (2.14) follows from (2.15)-(2.17).

Next, using the Lipschitz continuity of $A^{h}\left(e_{h}\right)$ in $V_{h}$ (see (1.14)), $A^{h}\left(e_{h}\right) 0=0,(2.6)$ and (2.13), we obtain

$$
\begin{aligned}
& \left|\left\langle A^{h}\left(e_{h}\right) u_{h}, v_{h}\right\rangle-\langle A(e) u, v\rangle\right| \\
& \quad \leq\left|\left\langle A^{h}\left(e_{h}\right) u_{h}, v_{h}-v\right\rangle\right|+\left|\left\langle A^{h}\left(e_{h}\right) u_{h}, v\right\rangle-\langle A(e) u, v\rangle\right| \rightarrow 0 .
\end{aligned}
$$

Consider the estimate

We may write

$$
\begin{aligned}
\left|\Phi_{h}\left(e_{h}\right) v_{h}-\Phi\left(e_{h}\right) v_{h}\right| & =\left|\sum_{E \subset \partial \Omega_{C}} \int_{E} \mathcal{F}_{h}\left(\left|v_{h}(\gamma)\right|-\left|v_{h}\right|\right) d s\right| \\
& \leq \mathcal{F}_{\max } \sum_{E} \int_{E}\left|v_{h}(\gamma)-v_{h}\right| d s .
\end{aligned}
$$

$$
\begin{aligned}
\left|v_{h}(\gamma)-v_{h}(s)\right| & \leq \frac{1}{2} \ell_{E}\left|\partial v_{h} / \partial s\right| \leq \frac{1}{2} \ell_{E}\left\|\operatorname{grad} v_{h}\left(T_{E}\right)\right\| \\
& \leq \ell_{E} C \varrho_{T}^{-1}\left|v_{h}\right|_{1, T_{E}} \leq \widetilde{C}\left|v_{h}\right|_{1, T_{E}},
\end{aligned}
$$

where $\ell_{E}=$ meas $E, T_{E}$ is the triangle adjacent to the edge $E$ and $\varrho_{T}$ is the radius of the largest circle inscribed in $T_{E}$.

Thus we obtain

$$
\begin{aligned}
& \sum_{E} \int_{E}\left|v_{h}(\gamma)-v_{h}\right| d s \leq \widetilde{C} \sum_{E} \ell_{E}\left|v_{h}\right|_{1, T_{E}} \\
& \leq \widetilde{C} h^{1 / 2}\left(\sum_{E} \ell_{E}\right)^{1 / 2}\left(\sum_{E}\left|v_{h}\right|_{1, T_{E}}^{2}\right)^{1 / 2} \leq \widetilde{C} h^{1 / 2}\left(\operatorname{meas} \partial \Omega_{C}\right)^{1 / 2}\left|v_{h}\right|_{1, \Omega} \rightarrow 0 .
\end{aligned}
$$

As a consequence,

$$
\Lambda_{1 h}:=\left|\Phi_{h}\left(e_{h}\right) v_{h}-\Phi\left(e_{h}\right) v_{h}\right| \rightarrow 0 .
$$

Since $v \in \mathcal{K}(H)$, we have

$$
\begin{aligned}
\Lambda_{2 h} & :=\left|\Phi\left(e_{h}\right) v_{h}-\Phi\left(e_{h}\right) v\right|=\left|\int_{\partial \Omega_{C}} \mathcal{F}_{h}\left(\left|v_{h}\right|-|v|\right) d s\right| \\
& \leq \mathcal{F}_{\max }\left(\operatorname{meas} \partial \Omega_{C}\right)^{1 / 2}\left\|v_{h}-v\right\|_{0, \partial \Omega_{C}} \rightarrow 0 .
\end{aligned}
$$

Finally, we may write

$$
\begin{aligned}
\Lambda_{3 h} & :=\left|\Phi\left(e_{h}\right) v-\Phi(e) v\right| \\
& =\left|\int_{\partial \Omega_{C}}\left(\mathcal{F}_{h}-\mathcal{F}\right)\right| v|d s| \leq\left\|\mathcal{F}_{h}-\mathcal{F}\right\|_{\infty} \int_{\partial \Omega_{C}}|v| d s \rightarrow 0 .
\end{aligned}
$$


Combining (2.19)-(2.21), we arrive at

$$
\left|\Phi_{h}\left(e_{h}\right) v_{h}-\Phi(e) v\right| \leq \Lambda_{1 h}+\Lambda_{2 h}+\Lambda_{3 h} \rightarrow 0 \quad \text { as } h \rightarrow 0+.
$$

In a parallel way, we can deduce that

$$
\left|\Phi_{h}\left(e_{h}\right) u_{h}-\Phi(e) u\right| \rightarrow 0 \quad \text { as } h \rightarrow 0+,
$$

using the boundedness of $\left\{u_{h}\right\}$ in $V$ and the compactness of the trace operator (cf. (2.20)).

On the basis of (1.1), (2.6) and the weak convergence (2.1), we obtain

$$
\begin{aligned}
& \left|\left[p, v_{h}-u_{h}\right]_{h}-\langle p, v-u\rangle_{0}\right| \\
& \quad \leq\left|\left[p, v_{h}-u_{h}\right]_{h}-\left\langle p, v_{h}-u_{h}\right\rangle_{0}\right|+\left|\left\langle p,\left(v_{h}-u_{h}\right)-(v-u)\right\rangle_{0}\right| \rightarrow 0 .
\end{aligned}
$$

Finally, it is easy to see that

$$
\begin{aligned}
& \left|\left\langle H_{h}, v_{h}-u_{h}\right\rangle_{0}-\langle H, v-u\rangle_{0}\right| \\
& \leq\left|\left\langle H_{h}-H, v_{h}-u_{h}\right\rangle_{0}\right|+\left|\left\langle H,\left(v_{h}-u_{h}\right)-(v-u)\right\rangle_{0}\right| \\
& \leq C\left\|H_{h}-H\right\|_{\infty}\left\|v_{h}-u_{h}\right\|_{0}+\|H\|_{0}\left\|v_{h}-v\right\|_{0}+\left|\left\langle H, u-u_{h}\right\rangle_{0}\right| \rightarrow 0 .
\end{aligned}
$$

Coming back to the variational inequality (1.4) and passing to limes inferior or limes superior as $h \rightarrow 0+$, we employ (2.12), (2.18), (2.22)-(2.25) to get

$$
\begin{aligned}
\langle A(e) u, u\rangle & \leq \liminf \left\langle A^{h}\left(e_{h}\right) u_{h}, u_{h}\right\rangle \leq \limsup \left\langle A^{h}\left(e_{h}\right) u_{h}, u_{h}\right\rangle \\
& \leq\langle A(e) u, v\rangle+\Phi(e) v-\Phi(e) u+\langle p-2 \omega H, u-v\rangle_{0}
\end{aligned}
$$

for all $v \in \mathcal{K}(H)$.

Thus $u$ is a solution of the inequality [3, (1.7)]. From the uniqueness of $u(e)$ we conclude that $u=u(e)$ and the whole sequence $\left\{u_{h}\left(e_{h}\right)\right\}$ tends to $u(e)$ weakly in $V$ as $h \rightarrow 0+$.

It remains to prove the strong convergence. We may set $v:=u$ in $(2.26)$ to obtain

$$
\lim \left\langle A^{h}\left(e_{h}\right) u_{h}, u_{h}\right\rangle=\langle A(e) u, u\rangle .
$$

Next, we have

$$
\begin{aligned}
& \left|\left\langle A(e) u_{h}, u_{h}\right\rangle-\left\langle A^{h}\left(e_{h}\right) u_{h}, u_{h}\right\rangle\right| \\
& \leq\left|\left\langle A(e) u_{h}, u_{h}\right\rangle-\left\langle A\left(e_{h}\right) u_{h}, u_{h}\right\rangle\right|+\left|\left\langle A\left(e_{h}\right) u_{h}, u_{h}\right\rangle-\left\langle A^{h}\left(e_{h}\right) u_{h}, u_{h}\right\rangle\right| \\
& \leq\left\|A(e) u_{h}-A\left(e_{h}\right) u_{h}\right\|_{*}\left\|u_{h}\right\|_{1}+C h\left\|u_{h}\right\|_{1}\left\|u_{h}\right\|_{0} \rightarrow 0,
\end{aligned}
$$

making use of (2.15) and (2.7). Thus

$$
\lim \left\langle A(e) u_{h}, u_{h}\right\rangle=\langle A(e) u, u\rangle
$$

follows from (2.27) and (2.28). 
Using (2.29), $[3,(2.15)]$ and $(2.9)$, we arrive at

$$
\begin{aligned}
\lim a\left(H ; u_{h}, u_{h}\right) & =\lim \left\langle A(e) u_{h}, u_{h}\right\rangle-\lim b\left(\mathcal{Z} ; u_{h}, u_{h}\right) \\
& =\langle A(e) u, u\rangle-b(\mathcal{Z} ; u, u)=a(H ; u, u) .
\end{aligned}
$$

The bilinear form $a(H ; \cdot, \cdot)$ can be taken for a scalar product in $V$ (see $[3,(2.16)])$. From $(2.30)$ and the weak convergence of $\left(u_{h}\right)$ we conclude that

$$
\lim a\left(H ; u_{h}-u, u_{h}-u\right)=0,
$$

which in turn implies that $u_{h} \rightarrow u$ in $V$.

Proposition 2.2. Let $e_{h} \in U_{\text {ad }}^{h}$ with $e_{h} \rightarrow e$ in $U$ as $h \rightarrow 0+$. Then

$$
\lim _{h \rightarrow 0+} \mathcal{L}_{J}^{h}\left(e_{h}, u_{h}\left(e_{h}\right)\right)=\mathcal{L}_{J}(e, u(e))
$$

for $J=\mathrm{DD}$, ISS, COM, TR, and

$$
\lim _{h \rightarrow 0+} \mathcal{L}_{W}\left(\varepsilon ; e_{h}, u_{h}\left(e_{h}\right)\right)=\mathcal{L}_{W}(\varepsilon ; e, u(e))
$$

for any $\varepsilon>0$.

Proof. Define $u:=u(e), u_{h}:=u_{h}\left(e_{h}\right)$. It is readily seen that

$$
\begin{aligned}
\left|\mathcal{L}_{\mathrm{DD}}^{h}\left(e_{h}, u_{h}\right)-\mathcal{L}_{\mathrm{DD}}(e, u)\right| & =\left|\int_{\Omega}\left(\left(u_{h}-z\right)^{2}-(u-z)^{2}\right) d x\right| \\
& \leq\left\|u_{h}-u\right\|_{0}\left\|u_{h}+u-2 z\right\|_{0} \rightarrow 0, \\
\left|\mathcal{L}_{\text {ISS }}^{h}\left(e_{h}, u_{h}\right)-\mathcal{L}_{\mathrm{ISS}}(e, u)\right| & =\left.|| u_{h}\right|_{1} ^{2}-|u|_{1}^{2}|\leq|\left|u_{h}\right|_{1}-|u|_{1} \mid \cdot\left(\left|u_{h}\right|_{1}+|u|_{1}\right) \\
& \leq C\left|u_{h}-u\right|_{1} \rightarrow 0 .
\end{aligned}
$$

Next, we have

$$
\begin{aligned}
& \left|\mathcal{L}_{\mathrm{COM}}^{h}\left(e_{h}, u_{h}\right)-\mathcal{L}_{\mathrm{COM}}(e, u)\right| \\
& \quad \leq\left|\mathcal{L}_{\mathrm{COM}}^{h}\left(e_{h}, u_{h}\right)-\mathcal{L}_{\mathrm{COM}}\left(e_{h}, u\right)\right|+\left|\mathcal{L}_{\mathrm{COM}}\left(e_{h}, u\right)-\mathcal{L}_{\mathrm{COM}}(e, u)\right| \equiv L_{1}+L_{2},
\end{aligned}
$$

where

$$
\begin{aligned}
L_{1} & \leq\left|\left[p, u_{h}\right]_{h}-\left\langle p, u_{h}\right\rangle_{0}\right|+\left|\left\langle p, u_{h}-u\right\rangle_{0}\right|+2 \omega\left|\left\langle H_{h}, u-u_{h}\right\rangle_{0}\right| \\
& \leq C h\left\|u_{h}\right\|_{1}+C_{1}\left\|u-u_{h}\right\|_{0} \rightarrow 0
\end{aligned}
$$

by (1.1) and Proposition 2.1.

Moreover,

$$
L_{2}=2 \omega\left|\left\langle H-H_{h}, u_{h}\right\rangle_{0}\right| \leq C\left\|H-H_{h}\right\|_{\infty}\left\|u_{h}\right\|_{0} \rightarrow 0,
$$

so that

$$
\mathcal{L}_{\mathrm{COM}}^{h}\left(e_{h}, u_{h}\right) \rightarrow \mathcal{L}_{\mathrm{COM}}(e, u) .
$$

Next, we may write

$$
\begin{aligned}
& \left|\mathcal{L}_{\mathrm{TR}}^{h}\left(e_{h}, u_{h}\right)-\mathcal{L}_{\mathrm{TR}}(e, u)\right| \\
& \quad \leq\left|\mathcal{L}_{\mathrm{TR}}^{h}\left(e_{h}, u_{h}\right)-\mathcal{L}_{\mathrm{TR}}\left(e_{h}, u\right)\right|+\left|\mathcal{L}_{\mathrm{TR}}\left(e_{h}, u\right)-\mathcal{L}_{\mathrm{TR}}(e, u)\right| \equiv M_{1}+M_{2} .
\end{aligned}
$$


Using also (2.7a) in the final step, we derive that

$$
\begin{aligned}
M_{1} & \leq\left|\left\langle H_{h} \operatorname{grad}\left(u_{h}-u\right), \operatorname{grad} \theta\right\rangle_{0}\right|+\left|\sum_{T \subset \bar{\Omega} \backslash \Omega^{*}}\left\langle\mathcal{Z}_{h}\left(\left[u_{h}(\gamma)\right]^{-}-[u]^{-}\right), \theta\right\rangle_{0, T}\right| \\
& \leq H_{\max } C\left\|u_{h}-u\right\|_{1}+\mathcal{Z}_{\max } \sum_{T} \int_{T}\left(\left|u_{h}(\gamma)-u_{h}\right|+\left|u_{h}-u\right|\right)|\theta| d x \\
& \leq H_{\max } C\left\|u_{h}-u\right\|_{1}+C_{1}\left(h\left\|u_{h}\right\|_{1}\|\theta\|_{0}+\sum_{T}\left\|u_{h}-u\right\|_{0, T}\|\theta\|_{0, T}\right) \rightarrow 0 .
\end{aligned}
$$

Next, we also have

$$
\begin{aligned}
M_{2} & \leq\left|\left\langle\left(H_{h}-H\right) \operatorname{grad} u, \operatorname{grad} \theta\right\rangle_{0}\right|+2 \omega\left|\left\langle H-H_{h}, \theta\right\rangle_{0}\right| \\
& +\left|\left\langle\left(\mathcal{Z}_{h}^{0}-\mathcal{Z}^{0}\right)[u]^{-}, \theta\right\rangle_{0}\right| \leq C\left(\left\|H_{h}-H\right\|_{\infty}+\left\|\mathcal{Z}_{h}^{0}-\mathcal{Z}^{0}\right\|_{\infty}\right)\|u\|_{1}\|\theta\|_{1} \rightarrow 0,
\end{aligned}
$$

so that

$$
\mathcal{L}_{\mathrm{TR}}^{h}\left(e_{h}, u_{h}\right) \rightarrow \mathcal{L}_{\mathrm{TR}}(e, u)
$$

Finally, we may write

$$
\begin{aligned}
\mid \mathcal{L}_{W}\left(\varepsilon, e_{h}, u_{h}\right)- & \mathcal{L}_{W}(\varepsilon ; e, u) \mid \\
& \leq\left|\left\langle\omega, H_{h}-H\right\rangle_{0}\right|+\varepsilon^{-1} \sum_{j=1}^{M}\left|\left[F_{j}\left(u_{h}\right)\right]^{+}-\left[F_{j}(u)\right]^{+}\right| \\
& \leq C\left\{\left\|H_{h}-H\right\|_{\infty}+\left\|u_{h}-u\right\|_{1}\left(\left\|u_{h}\right\|_{1}+\|u\|_{1}\right)\right\} \rightarrow 0,
\end{aligned}
$$

using an argument analogous to the proof of [3, Lemma 3.1].

Lemma 2.3. For any $e \equiv\{H, \mathcal{Z}, \mathcal{F}\} \in U_{\text {ad }}$ and any sequence $\{h\}$ with $h \rightarrow 0+$, there exists a sequence $\left\{e_{h}\right\}$ such that $e_{h} \equiv\left\{H_{h}, \mathcal{Z}_{h}, \mathcal{F}_{h}\right\} \in U_{\text {ad }}^{h}, \quad e_{h} \rightarrow e \quad$ in $U \equiv C(\bar{\Omega}) \times C\left(\bar{\Omega} \backslash \Omega^{*}\right) \times C\left(\bar{\partial}_{C}\right)$.

Proof. Let $\pi_{h} H$ denote the Lagrange linear interpolate of $H$ over the triangulation $\mathcal{T}_{h}$. Since $H \in W^{1, \infty}(\Omega)$, interpolation theory (see e.g. [1]) yields

$$
\left\|H-\pi_{h} H\right\|_{0, \infty} \leq C h\|H\|_{1, \infty} .
$$

Obviously, $H_{\min } \leq \pi_{h} H \leq H_{\max }$ everywhere. For any straight-line segment $\overline{P Q} \in T$ parallel to the $x_{i}$-axis and any triangle $T \subset \mathcal{T}_{h}$ we have

$$
\left|\partial \pi_{h} H / \partial x_{i}\right|=\ell^{-1}|H(Q)-H(P)| \leq \ell^{-1} \int_{P}^{Q}\left|\partial H / \partial x_{i}\right| \leq C_{i}^{H},
$$

where $\ell=|\overline{P Q}|$.

Analogous arguments hold for $\pi_{h} \mathcal{Z}$ and for $\pi_{h}^{0} \mathcal{F} \in X_{h}^{C}$, i.e., the Lagrange linear interpolate of $\mathcal{F}$ over the partition of $\partial \Omega_{C}$, generated by $\mathcal{T}_{h}$.

Now $e_{h}=\left\{\pi_{h} H, \pi_{h} \mathcal{Z}, \pi_{h}^{0} \mathcal{F}\right\}$ satisfies the conditions of the lemma. 
THEOREM 2.4. Let $\left\{e_{J}^{* h}\right\}, h \rightarrow 0+$, be a sequence of solutions to the Approximate Optimal Design Problem (1.6), $J=\mathrm{DD}$, ISS, COM, TR. Then there exists a subsequence $\left\{e_{J}^{* \widehat{h}}\right\} \subset\left\{e_{J}^{* h}\right\}$ such that

$$
\begin{gathered}
e_{J}^{* \widehat{h}} \rightarrow e_{J}^{*} \quad \text { in } U \equiv C(\bar{\Omega}) \times C\left(\bar{\Omega} \backslash \Omega^{*}\right) \times C\left(\overline{\partial \Omega}_{C}\right), \\
u_{\widehat{h}}\left(e_{J}^{* \widehat{h}}\right) \rightarrow u\left(e_{J}^{*}\right) \quad \text { in } V,
\end{gathered}
$$

where $e_{J}^{*}$ is a solution of the Optimal Design Problem [3, one of (1.14)(1.17)]. The limit of each subsequence of $\left\{e_{J}^{* h}\right\}$, converging in $U$, is a solution of the latter problem and an analogue of (2.32) holds.

Proof. Since each $U_{\text {ad }}^{h} \subset U_{\text {ad }}$ and $U_{\text {ad }}$ is compact in $U$, there exists a subsequence $\left\{e_{J}^{* \widehat{h}}\right\}, \widehat{h} \rightarrow 0+$, such that $(2.31)$ holds. Consider an $e \in U_{\mathrm{ad}}$. By Lemma 2.3, there exists a sequence of $e_{\widehat{h}} \in U_{\text {ad }}^{\widehat{h}}$ such that $e_{\widehat{h}} \rightarrow e$ in $U$ as $\widehat{h} \rightarrow 0+$. By definition, we have

$$
\mathcal{L}_{J}^{\widehat{h}}\left(e_{J}^{* \widehat{h}}, u_{\widehat{h}}\left(e_{J}^{* \widehat{h}}\right)\right) \leq \mathcal{L}_{J}^{\widehat{h}}\left(e_{\widehat{h}}, u_{\widehat{h}}\left(e_{\widehat{h}}\right)\right) .
$$

Letting $\widehat{h} \rightarrow 0+$ and applying Proposition 2.2 to both sides of this inequality, we arrive at

$$
\mathcal{L}_{J}\left(e_{J}^{*}, u\left(e_{J}^{*}\right)\right) \leq \mathcal{L}_{J}(e, u(e)),
$$

so that $e_{J}^{*}$ is a solution of the original Optimal Design Problem. Making use of Proposition 2.1, we obtain (2.32). This line of thought may be repeated for any uniformly convergent subsequence of $\left\{e_{J}^{* h}\right\}$.

TheOREm 2.5. Let $\left\{e_{h}^{\varepsilon}\right\}, h \rightarrow 0+$, be a sequence of solutions of the Approximate Weight Minimization Problem (1.23). Then there exists a subsequence $\left\{e_{\widehat{h}}^{\varepsilon}\right\} \subset\left\{e_{h}^{\varepsilon}\right\}$ such that

$$
e_{\widehat{h}}^{\varepsilon} \rightarrow e_{\varepsilon} \quad \text { in } U
$$

where $e_{\varepsilon}$ is a solution of the penalized optimization problem [3, (3.1)].

Proof. Analogous to that of Theorem 2.4.

3. Approximate reliable solutions. We shall introduce approximations of the method of reliable solution (alias worst scenario method), which has been introduced in [3, Section 4] for problems with some uncertain input data. In contrast with the previous sections, we keep the half-thickness $H(x)$ fixed, $H \in C^{(0), 1}(\bar{\Omega}), H>0$ everywhere and $O_{i} \geq \max _{x \in \bar{\Omega}} H(x), 1 \leq i \leq N$ (see $[3,(1.1)])$. On the other hand, we allow the loading function $p$ to vary in the set $U_{\mathrm{ad}}^{p}$.

Here we use again the finite element spaces $X_{h}, V_{h}$, and the sets $U_{\text {ad }}^{\mathcal{Z} h}$, $U_{\text {ad }}^{\mathcal{F} h}$, but we introduce a new set $U_{\text {ad }}^{p h}=U_{\text {ad }}^{p} \cap X_{h}$. Assume that $p_{0} \in X_{h_{0}}$ for some triangulation $\mathcal{T}_{h_{0}}$. 
Hence we have to assume that the triangulations $\mathcal{T}_{h}$ are consistent also with the boundaries $\partial \Omega_{m}, m=1, \ldots, M$, which play a role in the definition of $U_{\mathrm{ad}}^{p}$, and with the boundaries of $G_{j}$, which appear in the definition of $\Phi_{1}$ and $\Phi_{2}$. Then we define

$$
U_{\mathrm{ad}}^{h}=U_{\mathrm{ad}}^{p h} \times U_{\mathrm{ad}}^{\mathcal{Z} h} \times U_{\mathrm{ad}}^{\mathcal{F} h}
$$

and consider approximate input data $e_{h}=\left\{p_{h}, \mathcal{Z}_{h}, \mathcal{F}_{h}\right\} \in U_{\text {ad }}^{h}$.

Instead of the criterions $\Phi_{i}, i=1,2,3$, we introduce

$$
\begin{aligned}
\Phi_{1}^{h}\left(v_{h}\right)= & \max _{1 \leq j \leq J}\left(\operatorname{meas} G_{j}\right)^{-1} \sum_{T \subset G_{j}}\left|v_{h}(\gamma)\right| \operatorname{meas} T, \\
\Phi_{2}^{h}\left(v_{h}\right)= & \Phi_{2}\left(v_{h}\right)=\max _{1 \leq j \leq J}\left(\operatorname{meas} G_{j}\right)^{-1} \int_{G_{j}}\left|\operatorname{grad} v_{h}\right|^{2} d x, \\
\Phi_{3}^{h}\left(e_{h}, v_{h}\right)= & \left\langle H \operatorname{grad} v_{h}, \operatorname{grad} \varphi\right\rangle_{0}+\left\langle 2 \omega H-p_{h}, \varphi\right\rangle_{0} \\
& +\sum_{T \subset \bar{\Omega} \backslash \Omega^{*}}\left\langle\mathcal{Z}_{h}\left[v_{h}(\gamma)\right]^{-}, \varphi\right\rangle_{0, T}, \quad \varphi \in H_{0}^{1}(\Omega) \cap X_{h} .
\end{aligned}
$$

We solve the following approximate maximization problems: find

$$
e_{i}^{* h}=\arg \max _{e_{h} \in U_{\mathrm{ad}}^{h}} \Phi_{i}^{h}\left(e_{h}, u_{h}\left(e_{h}\right)\right), \quad i=1,2,3,
$$

where $u_{h}\left(e_{h}\right)$ denotes the solution of the Approximate State Problem (1.4) for the input data $e_{h} \equiv\left\{p_{h}, \mathcal{Z}_{h}, \mathcal{F}_{h}\right\} \in U_{\text {ad }}^{h}$, i.e., $u_{h}\left(e_{h}\right) \in \mathcal{K}_{h}(H)$ such that

$$
\begin{aligned}
& a\left(H ; u_{h}\left(e_{h}\right), v_{h}-u_{h}\left(e_{h}\right)\right)+b_{h}\left(\mathcal{Z}_{h} ; u_{h}\left(e_{h}\right), v_{h}-u_{h}\left(e_{h}\right)\right) \\
& \quad+\Phi_{h}\left(e_{h}\right)\left(v_{h}\right)-\Phi_{h}\left(e_{h}\right)\left(u_{h}\left(e_{h}\right)\right) \geq\left\langle p_{h}-2 \omega H, v_{h}-u_{h}\left(e_{h}\right)\right\rangle_{0}
\end{aligned}
$$

for all $v_{h} \in \mathcal{K}_{h}(H)$.

THEOREM 3.1. (i) The problem (3.2) has a unique solution $u_{h}\left(e_{h}\right)$ for any $e_{h} \in U_{\mathrm{ad}}^{h}$ and any $h$ sufficiently small.

(ii) The approximate maximization problem $\left(3.1_{i}\right), i=1,2,3$, has at least one solution for any $h$ sufficiently small.

Proof. The argument is analogous to that of Theorem 1.1. Let us verify the assumptions of [3, Theorem 2.1], where we set $\mathcal{K}(e):=\mathcal{K}_{h}(H)$, $\left\langle f, v_{h}\right\rangle=-2 \omega\left\langle H, v_{h}\right\rangle_{0},\left\langle B e, v_{h}\right\rangle=\left\langle p_{h}, v_{h}\right\rangle_{0}, U_{\text {ad }}:=U_{\text {ad }}^{h}, e:=e_{h}, V:=V_{h}$, $A(e):=A^{h}\left(e_{h}\right)$,

$$
\begin{aligned}
\left\langle A^{h}\left(e_{h}\right) v_{h}, w_{h}\right\rangle & :=a\left(H ; v_{h}, w_{h}\right)+b_{h}\left(\mathcal{Z}_{h} ; v_{h}, w_{h}\right), \\
\Phi(e)\left(v_{h}\right) & :=\Phi_{h}\left(e_{h}\right)\left(v_{h}\right)=\sum_{E \subset \partial \Omega_{C}} \int_{E} \mathcal{F}_{h}\left|v_{h}(\gamma)\right| d s+I_{\mathcal{K}_{h}(H)}\left(v_{h}\right) .
\end{aligned}
$$

Then Lemma 1.3 holds and Lemma 1.4 can be proved by nearly the same (simpler) argument. Instead of Lemma 1.5 we prove the following 
LEMMA 3.2. Let $e_{h n} \rightarrow e_{h}, e_{h n} \in U_{\mathrm{ad}}^{h}$ and $v_{h n} \rightarrow v_{h}, v_{h n} \in V_{h}$, as $n \rightarrow \infty$. Then

$$
\lim _{n \rightarrow \infty} \Phi_{i}^{h}\left(e_{h n}, v_{h n}\right)=\Phi_{i}^{h}\left(e_{h}, v_{h}\right), \quad i=1,2,3 .
$$

Proof. We may write

$$
\begin{aligned}
\lim _{n \rightarrow \infty} \Phi_{1}^{h}\left(v_{h n}\right) & =\lim \max _{1 \leq j \leq J}\left(\operatorname{meas} G_{j}\right)^{-1} \sum_{T \subset G_{j}}\left|v_{h n}(\gamma)\right| \operatorname{meas} T \\
& =\max _{j} \lim _{n \rightarrow \infty} \psi_{j}\left(v_{h n}\right)=\max _{j} \psi_{j}\left(v_{h}\right)=\Phi_{1}^{h}\left(v_{h}\right),
\end{aligned}
$$

since

$$
\begin{aligned}
& \left|\sum_{T \subset G_{j}}\right| v_{h n}(\gamma) \mid \text { meas } T-\sum_{T \subset G_{j}}\left|v_{h}(\gamma)\right| \text { meas } T \mid \\
& \quad \leq \sum_{T \subset G_{j}}\left|v_{h n}(\gamma)-v_{h}(\gamma)\right| \text { meas } T \leq \sum_{T \subset G_{j}}\left\|v_{h n}-v_{h}\right\|_{\infty, T} \text { meas } T \\
& \quad \leq \sum_{T \subset G_{j}} C\left\|v_{h n}-v_{h}\right\|_{0, T}(\text { meas } T)^{1 / 2} \leq C\left\|v_{h n}-v_{h}\right\|_{0, G_{j}} \text { meas } G_{j} \rightarrow 0 .
\end{aligned}
$$

Here we have used the inequality (1.15) in the final step.

Second, we have

$$
\left|\int_{G_{j}}\left(\left|\operatorname{grad} v_{h n}\right|^{2}-\left|\operatorname{grad} v_{h}\right|^{2}\right) d x\right| \leq\left\|v_{h n}-v_{h}\right\|_{1}\left(\left\|v_{h n}\right\|_{1}+\left\|v_{h}\right\|_{1}\right) \rightarrow 0,
$$

so that

$$
\begin{aligned}
\lim _{n \rightarrow \infty} \Phi_{2}^{h}\left(v_{h n}\right) & =\lim _{n \rightarrow \infty} \max _{1 \leq j \leq J}\left(\operatorname{meas} G_{j}\right)^{-1} \int_{G_{j}}\left|\operatorname{grad} v_{h n}\right|^{2} d x \\
& =\max _{j} \lim _{n \rightarrow \infty}(\ldots)=\Phi_{2}^{h}\left(v_{h}\right) .
\end{aligned}
$$

Third, we may write

$$
\begin{aligned}
\left|\Phi_{3}^{h}\left(e_{h n}, v_{h n}\right)-\Phi_{3}^{h}\left(e_{h}, v_{h}\right)\right| \leq & \left|\Phi_{3}^{h}\left(e_{h n}, v_{h n}\right)-\Phi_{3}^{h}\left(e_{h}, v_{h n}\right)\right| \\
& +\left|\Phi_{3}^{h}\left(e_{h}, v_{h n}\right)-\Phi_{3}^{h}\left(e_{h}, v_{h}\right)\right| \equiv L_{1}+L_{2},
\end{aligned}
$$

and using (1.15) again,

$$
\begin{gathered}
L_{1} \leq\left|\left\langle p_{h n}-p_{h}, \varphi\right\rangle_{0}\right|+\sum_{T}\left|\left\langle\left(\mathcal{Z}_{h n}-\mathcal{Z}_{h}\right)\left[v_{h n}(\gamma)\right]^{-}, \varphi\right\rangle_{0, T}\right| \\
\leq C\left(\left\|p_{h n}-p_{h}\right\|_{0}+\left\|\mathcal{Z}_{h n}-\mathcal{Z}_{h}\right\|_{\infty}\left\|v_{h n}\right\|_{0}\right) \rightarrow 0, \\
L_{2} \leq\left|\left\langle H \operatorname{grad}\left(v_{h n}-v_{h}\right), \operatorname{grad} \varphi\right\rangle_{0}\right|+\sum_{T}\left|\left\langle\mathcal{Z}_{h}\left(\left[v_{h n}(\gamma)\right]^{-}-\left[v_{h}(\gamma)\right]^{-}\right), \varphi\right\rangle_{0, T}\right| \\
\leq C\left\|v_{h n}-v_{h}\right\|_{1}+\mathcal{Z}_{\max } \sum_{T}\left\|v_{h n}-v_{h}\right\|_{0, T} \cdot\|\varphi\|_{0, T} \rightarrow 0 .
\end{gathered}
$$


As a consequence,

$$
\lim _{n \rightarrow \infty} \Phi_{3}^{h}\left(e_{h n}, v_{h n}\right)=\Phi_{3}^{h}\left(e_{h}, v_{h}\right) .
$$

Finally, the existence of solutions of the problems $\left(3.1_{i}\right)$ follows if we set $\mathcal{L} \equiv-\Phi_{i}^{h}$.

4. Convergence results. Let us study the convergence of finiteelement approximations when the mesh size tends to zero. First of all, we have to establish the following

Proposition 4.1. Let $e_{h} \in U_{\text {ad }}^{h}$ with $e_{h} \rightarrow e$ in $U$ as $h \rightarrow 0+$. Then

$$
u_{h}\left(e_{h}\right) \rightarrow u(e) \quad \text { in } V \text { as } h \rightarrow 0+.
$$

Proof. The proof is analogous to that of Proposition 2.1. We can insert $v_{h}=0$ in the inequality (3.2) to get the boundedness of $u_{h}:=u_{h}\left(e_{h}\right)$ as $h \rightarrow 0+$. In proving that the weak limit of a subsequence $\left\{u_{h}\right\}$ belongs to $\mathcal{K}(H)$, we substitute $H_{h}:=\pi_{h} H$, i.e., the linear Lagrange interpolate of $H$ over the triangulation $\mathcal{T}_{h}$, and use the fact that

$$
\left\|\pi_{h} H-H\right\|_{0, \Omega_{i}^{*}} \rightarrow 0 \quad \text { as } h \rightarrow 0+
$$

(cf. the proof of Lemma 2.3).

We derive (2.7)-(2.18), (2.22), (2.23). Instead of (2.24), (2.25) we obtain

$$
\begin{aligned}
& \quad\left|\left\langle p_{h}-2 \omega H, v_{h}-u_{h}\right\rangle_{0}-\langle p-2 \omega H, v-u\rangle_{0}\right| \\
& \leq C\left\{\left\|v_{h}-v\right\|_{0}+\left\|u_{h}-u\right\|_{0}+\left\|p_{h}-p\right\|_{\infty}\right\} \rightarrow 0 .
\end{aligned}
$$

Passing to limes inferior or limes superior in the inequality (3.2) and employing (2.12), (2.18), (2.22), (2.23) and (4.1), we arrive at (2.26), so that $u$ satisfies the inequality $[3,(1.7)]$. As a consequence, the whole sequence $\left\{u_{h}\left(e_{h}\right)\right\}$ tends to $u(e)$ weakly in $V$ as $h \rightarrow 0+$.

The proof of strong convergence is the same as in the proof of Proposition 2.1 .

Proposition 4.2. Let $e_{h} \in U_{\mathrm{ad}}^{h}$ with $e_{h} \rightarrow e$ in $U$ as $h \rightarrow 0+$. Then

$$
\lim _{h \rightarrow 0+} \Phi_{i}^{h}\left(e_{h}, u_{h}\left(e_{h}\right)\right)=\Phi_{i}(e, u(e)), \quad i=1,2,3 .
$$

Proof. For $u_{h}:=u_{h}\left(e_{h}\right)$ and $u:=u(e)$ we may write

$$
\begin{aligned}
&\left|\sum_{T \subset G_{j}}\right| u_{h}(\gamma) \mid \operatorname{meas} T-\int_{G_{j}}|u| d x \mid \leq \sum_{T \subset G_{j} T} \int_{T}\left(\left|u_{h}(\gamma)-u_{h}\right|+\left|u_{h}-u\right|\right) d x \\
& \leq\left(h\left\|u_{h}\right\|_{1, G_{j}}+\left\|u_{h}-u\right\|_{0, G_{j}}\right) \text { meas } G_{j} \rightarrow 0 \quad \text { as } h \rightarrow 0,
\end{aligned}
$$


by Proposition 4.1. As a consequence,

$$
\begin{aligned}
\lim _{h \rightarrow 0+} \Phi_{1}^{h}\left(u_{h}\right) & =\lim _{h \rightarrow 0} \max _{1 \leq j \leq J}\left(\operatorname{meas} G_{j}\right)^{-1} \sum_{T \subset G_{j}}\left|u_{h}(\gamma)\right| \operatorname{meas} T \\
& =\max _{j \leq J} \lim _{h \rightarrow 0}(\ldots)=\max _{j \leq J}\left(\operatorname{meas} G_{j}\right)^{-1} \int_{G_{j}}|u| d x=\Phi_{1}(u) .
\end{aligned}
$$

Since

$$
\left.\left|\int_{G_{j}}\right| \operatorname{grad} u_{h}\right|^{2} d x-\int_{G_{j}}|\operatorname{grad} u|^{2} d x|\leq C| u_{h}-\left.u\right|_{1, G_{j}} \rightarrow 0,
$$

we have

$$
\begin{aligned}
\lim _{h \rightarrow 0+} \Phi_{2}^{h}\left(u_{h}\right) & =\lim _{h \rightarrow 0+} \max _{1 \leq j \leq J}\left(\operatorname{meas} G_{j}\right)^{-1} \int_{G_{j}}\left|\operatorname{grad} u_{h}\right|^{2} d x \\
& =\max _{j \leq J} \lim _{h \rightarrow 0+}(\ldots)=\Phi_{2}(u) .
\end{aligned}
$$

Third, we may write

$$
\begin{aligned}
\left|\Phi_{3}^{h}\left(e_{h}, u_{h}\right)-\Phi_{3}(e, u)\right| & \leq\left|\Phi_{3}^{h}\left(e_{h}, u_{h}\right)-\Phi_{3}\left(e_{h}, u\right)\right|+\left|\Phi_{3}\left(e_{h}, u\right)-\Phi_{3}(e, u)\right| \\
& =M_{1}+M_{2},
\end{aligned}
$$

where

$$
\begin{aligned}
M_{1} & \leq\left|\left\langle H \operatorname{grad}\left(u_{h}-u\right), \operatorname{grad} \varphi\right\rangle_{0}\right|+\sum_{T \subset \bar{\Omega} \backslash \Omega^{*}}\left|\left\langle\mathcal{Z}_{h}\left(\left[u_{h}(\gamma)\right]^{-}-[u]^{-}\right), \varphi\right\rangle_{0, T}\right| \\
& \leq C\left\|u_{h}-u\right\|_{1}+C_{1}\left(h\left\|u_{h}\right\|_{1}+\left\|u_{h}-u\right\|_{0}\right)\|\varphi\|_{0} \rightarrow 0
\end{aligned}
$$

(cf. the proof of Proposition 2.2 for $\mathcal{L}_{\mathrm{TR}}^{h}$ ), and

$$
\begin{aligned}
M_{2} & \leq\left|\int_{\Omega \backslash \Omega^{*}}\left(\mathcal{Z}_{h}-\mathcal{Z}\right)[u]^{-} \varphi d x\right|+\left|\left\langle p-p_{h}, \varphi\right\rangle_{0}\right| \\
& \leq C\left(\left\|\mathcal{Z}_{h}-\mathcal{Z}\right\|_{\infty}+\left\|p-p_{h}\right\|_{\infty}\right) \rightarrow 0 .
\end{aligned}
$$

As a consequence, we obtain $\lim _{h \rightarrow 0} \Phi_{3}^{h}\left(e_{h}, u_{h}\right)=\Phi_{3}(e, u)$.

Lemma 4.3. For any $e \equiv\{p, \mathcal{Z}, \mathcal{F}\} \in U_{\text {ad }}$ and any sequence $\{h\}, h \rightarrow 0+$, there exists a sequence $\left\{e_{h}\right\}$ such that $e_{h} \equiv\left\{p_{h}, \mathcal{Z}_{h}, \mathcal{F}_{h}\right\} \in U_{\text {ad }}^{h}$ and $e_{h} \rightarrow e$ in $U=\left(\prod_{m=1}^{M} C\left(\bar{\Omega}_{m}\right)\right) \times C\left(\bar{\Omega} \backslash \Omega^{*}\right) \times C\left(\overline{\partial \Omega}_{C}\right)$.

Proof. Consider the restriction $p_{m}=p \mid \Omega_{m}$ of any $p \in U_{\mathrm{ad}}^{p}$ and define $p_{h}=\pi_{h} p_{\varepsilon}$, where $\pi_{h}$ is the linear Lagrange interpolation over $\mathcal{T}_{h}$ and

$$
p_{\varepsilon}=\varepsilon p_{0}+(1-\varepsilon) p_{m}, \quad x \in \Omega_{m},
$$

where $\varepsilon$ is a real parameter, $0<\varepsilon<1$. We have

$$
\begin{aligned}
\left\|\partial p_{\varepsilon} / \partial x_{i}\right\|_{\infty, \Omega_{m}} & \leq \varepsilon\left\|\partial p_{0} / \partial x_{i}\right\|_{\infty}+(1-\varepsilon)\left\|\partial p_{m} / \partial x_{i}\right\|_{\infty} \\
& \leq C_{2}, \quad i=1,2
\end{aligned}
$$


by definitions of $U_{\text {ad }}$ and $p_{0}$. Since

$$
\left\|p_{\varepsilon}\right\|_{\infty, \Omega_{m}} \leq \varepsilon\left\|p_{0}\right\|_{\infty}+(1-\varepsilon)\left\|p_{m}\right\|_{\infty} \leq \max \left\{\left\|p_{0}\right\|_{\infty},\left\|p_{m}\right\|_{\infty}\right\} \equiv C_{3},
$$

we obtain

$$
\left\|p_{\varepsilon}\right\|_{1, \infty, \Omega_{m}} \leq C_{3}+2 C_{1} \equiv C_{4}
$$

for all $\varepsilon$. Using the estimate

$$
\left\|q-\pi_{h} q\right\|_{\infty, \Omega_{m}} \leq C h\|q\|_{1, \infty, \Omega_{m}}
$$

we may write

$$
\begin{aligned}
\left\|p_{h}-p_{0}\right\|_{\infty, \Omega_{m}} & \leq\left\|\pi_{h} p_{\varepsilon}-p_{\varepsilon}\right\|_{\infty}+\left\|p_{\varepsilon}-p_{0}\right\|_{\infty} \\
& \leq C C_{4} h+(1-\varepsilon)\left\|p_{m}-p_{0}\right\|_{\infty} \\
& \leq C C_{4} h+(1-\varepsilon) C_{1} \leq C_{1}
\end{aligned}
$$

if

$$
C C_{4} h \leq C_{1} \varepsilon .
$$

Let $\overline{P Q} \subset T \subset \bar{\Omega}_{m}$ be a straight-line segment of length $\ell$, parallel to the $x_{i}$-axis. Then

$$
\left|\partial \pi_{h} p_{\varepsilon} / \partial x_{i}\right|=\left|\ell^{-1} \int_{P}^{Q} \frac{\partial p_{\varepsilon}}{\partial x_{i}} d x_{i}\right| \leq \ell^{-1} \int_{P}^{Q}\left|\partial p_{\varepsilon} / \partial x_{i}\right| d x_{i} \leq C_{2}
$$

by (4.2), so that

$$
\left\|\partial p_{h} / \partial x_{i}\right\|_{\infty, \Omega_{m}} \leq C_{2}, \quad i=1,2
$$

Next, we have

$$
\begin{aligned}
\left\|p_{h}-p_{m}\right\|_{\infty, \Omega_{m}} & \leq\left\|\pi_{h} p_{\varepsilon}-p_{\varepsilon}\right\|_{\infty}+\left\|p_{\varepsilon}-p_{m}\right\|_{\infty} \\
& \leq C C_{4} h+\varepsilon\left\|p_{0}-p_{m}\right\|_{\infty} \\
& \leq C C_{4} h+\varepsilon C_{1} \rightarrow 0 \quad \text { as } h \rightarrow 0+\text { and } \varepsilon \rightarrow 0+.
\end{aligned}
$$

Combining (4.3)-(4.6), we can find a sequence $\left\{p_{h}\right\}, h \rightarrow 0+$, such that $p_{h} \in U_{\mathrm{ad}}^{p h}$ and $p_{h} \rightarrow p$ in $\prod_{m=1}^{M} C\left(\bar{\Omega}_{m}\right)$.

The components $\mathcal{Z}_{h}$ and $\mathcal{F}_{h}$ can be defined as linear Lagrange interpolates of $\mathcal{Z}$ and $\mathcal{F}$, respectively (cf. the proof of Lemma 2.3).

TheOREM 4.4. Let $\left\{e_{i}^{* h}\right\}, h \rightarrow 0+$, be a sequence of solutions of the approximate maximization problem $\left(3.1_{i}\right), i=1,2,3$. Then there exists a subsequence $\left\{e_{i}^{* \widehat{h}}\right\} \subset\left\{e_{i}^{* h}\right\}$ such that

$$
\begin{aligned}
e_{i}^{* \widehat{h}} & \rightarrow e_{i}^{*} \quad \text { in } U, \\
u_{\widehat{h}}\left(e_{i}^{* \widehat{h}}\right) & \rightarrow u\left(e_{i}^{*}\right) \quad \text { in } V, \\
\Phi_{i}^{\widehat{h}}\left(e_{i}^{* \widehat{h}}, u_{\widehat{h}}\left(e_{i}^{* \widehat{h}}\right)\right) & \rightarrow \Phi_{i}\left(e_{i}^{*}, u\left(e_{i}^{*}\right)\right),
\end{aligned}
$$


where $e_{i}^{*}$ is a solution of the maximization problem $(4.1)_{i}$ of [3]. The limit of each subsequence of $\left\{e_{i}^{* h}\right\}$, converging in $U$, is a solution of the problem $(4.1)_{i}$ and the analogues of (4.8), (4.9) hold.

Proof. Analogous to that of Theorem 2.4. Instead of Proposition 2.2 and Lemma 2.3, we employ Proposition 4.2 and Lemma 4.3. Proposition 2.1 is replaced by Proposition 4.1.

Acknowledgments. The first author thankfully acknowledges the support of the Grant Agency of the Czech Republic under grant 201/98/0528 and of the Ministry of Education, Youth and Sports under grant OK-407.

\section{References}

[1] P. G. Ciarlet, The Finite Element Method for Elliptic Problems, North-Holland, 1978.

[2] R. Glowinski, Lectures on Numerical Methods for Non-linear Variational Problems, Tata Inst. Fund. Res. and Springer, 1980.

[3] I. Hlaváček and J. Lovíšek, Control in obstacle-pseudoplate problems with friction on the boundary. Optimal design and problems with uncertain data, Appl. Math. (Warsaw) 28 (2001), 407-426.

[4] J. Nečas, Les méthodes directes en théorie des équations elliptiques, Academia, 1967, and Masson, 1967.

Mathematical Institute

Academy of Sciences of the Czech Republic

Žitná 25

CZ-115 67 Praha 1, Czech Republic

E-mail: hlavacek@math.cas.cz

Institute of Computer Science

Pod vodárenskou věží 2

CZ-182 07 Praha 8, Czech Republic
Faculty of Civil Engineering Slovak Technical University Radlinského 11

SK-813 68 Bratislava, Slovak Republic E-mail: bock@kmat.elf.stuba.sk 